\title{
Diagnosis-to-surgery interval and survival for different histologies of stage I-IIA lung cancer
}

\author{
Lu Zhang ${ }^{1}$, Mei-Chin Hsieh ${ }^{2}$, Lior Rennert ${ }^{1}$, Paige Neroda ${ }^{1}$, Xiao-Cheng $\mathbf{W u}^{2}$, Chindo Hicks ${ }^{3}$, Jiande $\mathbf{W u}^{3}$, \\ Ronald Gimbel $^{1}$
}

${ }^{1}$ Department of Public Health Sciences, Clemson University, Clemson, SC, USA; ${ }^{2}$ Louisiana Tumor Registry, School of Public Health Sciences, Louisiana State University Health Sciences Center, New Orleans, LA, USA; ${ }^{3}$ Genetic Department, School of Medicine, Louisiana State University Health Sciences Center, New Orleans, LA, USA

Contributions: (I) Conception and design: All authors; (II) Administrative support: L Zhang, XC Wu, R Gimbel; (III) Provision of study materials or patients: L Zhang; (IV) Collection and assembly of data: L Zhang; (V) Data analysis and interpretation: L Zhang; (VI) Manuscript writing: All authors; (VII) Final approval of manuscript: All authors.

Correspondence to: Lu Zhang, PhD. 513 Edwards Hall, Clemson University, Clemson, SC 29634, USA. Email: Lz3@clemson.edu.

Background: Guidelines on timeliness of lung cancer surgery are inconsistent. Lung cancer histologic subtypes have different prognosis and treatment. It is important to understand the consequences of delayed surgery for each lung cancer histologic subtype. This study aimed to examine the association between diagnosis-to-surgery time interval and survival for early stage lung cancer and selected histologic subtypes.

Methods: Patients diagnosed with stage I-IIA lung cancer between 2004 and 2015 receiving definitive surgery and being followed up until Dec. 31, 2018, were identified from Surveillance, Epidemiology, and End Results database. Histologic subtypes included adenocarcinoma, squamous or epidermoid carcinoma, bronchioloalveolar carcinoma, large cell carcinoma, adenosquamous carcinoma, carcinoid carcinoma, and small cell carcinoma. Diagnosis-to-surgery interval was treated as multi-categorical variables $(<1,1-2,2-3$, and $\geq 3$ months) and binary variables ( $\geq 1 v s .<1$ month, $\geq 2$ vs. $<2$ months, and $\geq 3 v s .<3$ months). Outcomes included cancer-specific and overall survival. Covariates included age at diagnosis, sex, race, marital status, tumor size, grade, surgery type, chemotherapy, radiotherapy, and study period. Kaplan-Meier survival curves and Cox proportional hazards regression models were applied to examine the survival differences.

Results: With a median follow-up time of 51 months, a total of 40,612 patients were analyzed, including $40.1 \%$ adenocarcinoma and $24.5 \%$ squamous or epidermoid carcinoma. The proportion of patients receiving surgery $<1,1-2,2-3$, and $\geq 3$ months from diagnosis were $34.2 \%, 33.9 \%, 19.8 \%$, and $12.1 \%$, respectively. Delayed surgery was associated with worse cancer-specific and overall survival for all lung cancers, adenocarcinoma, squamous or epidermoid, bronchioloalveolar, and large cell carcinoma (20-40\% increased risk). Dose-dependent effects (longer delay, worse survival) were observed in all lung cancers, adenocarcinoma, and squamous and epidermoid carcinoma. No significant association between surgery delay and survival was observed in adenosquamous, carcinoid, and small cell carcinoma.

Conclusions: Our findings support the guidelines of undertaking surgery within 1 month from diagnosis in patients with stage I-IIA lung cancer. The observed dose-dependent effects emphasize the clinical importance of early surgery. Future studies with larger sample size of less frequent histologic subtypes are warranted to provide more evidence for histology-specific lung cancer treatment guidelines.

Keywords: Lung cancer; histologic subtype; surgery delay; survival

Submitted Mar 02, 2021. Accepted for publication May 20, 2021.

doi: $10.21037 /$ tlcr-21-168

View this article at: https://dx.doi.org/10.21037/tlcr-21-168 


\section{Introduction}

Lung cancer is the deadliest cancer in the United States (U.S.) (1). In 2017, lung cancer caused more deaths than breast, prostate, colorectal, and brain cancers combined (1). A large randomized controlled trial found that annual screening can decrease lung cancer mortality by $20 \%$ in the high risk population (2), which indicates the effect of early detection and timely treatment on lung cancer survival. Timely treatment has been associated with improved survival for many types of cancer, especially for the early stage diseases (3-6). However, there is a lack of consensus on the impact of timely care on lung cancer survival. Although surgery is the initial treatment recommended to the operable diseases $(7,8)$, the major clinical guidelines on the timing of lung cancer surgery are inconsistent. Rand Corporation recommended lung cancer surgery within 6 weeks from diagnosis in 2000 and did not update the guidelines since then (9). American College of Chest Physicians (ACCP) recommended surgery within 4-8 weeks of referral in 2003 guidelines (10), but in its most recent guidelines from 2013, only 'interventions to improve timeliness should be developed locally by addressing barriers to providing timely care that are specific to the local setting' was recommended (11). British Thoracic Surgery (BTS) suggested a maximum of 8 weeks from respiratory specialist consultation to surgery in 1998 (12), however, no specific recommendations on the timing of treatment was provided in its 2019 guidelines (13). Danish Lung Cancer Group and Registry recommended less than 14 days from diagnosis to treatment (14). The reason for the inconsistency in the clinical guidelines could be the conflicting findings from the previous research. Thus, well designed epidemiological studies with more contemporary data and larger sample size is needed to describe the association between timely surgery and lung cancer survival.

Intensive work has been done to differentiate histologic subtypes of lung cancer, which can be used to predict prognosis and personalize treatment. Generally, two broad classes of lung cancer are non-small cell lung cancer (NSCLC) and small cell lung cancer (SCLC). NSCLC is a group of several histologies, representing about $85 \%$ of all lung cancer cases. The recommended initial treatment for early stage NSCLC is surgery (7). SCLC has a higher tumor growth rate and worse prognosis than NSCLC (15). Surgical resection is also recommended to operable SCLC, however, most SCLC has spread at diagnosis, for which the mainstay treatment is chemotherapy (8). In the past two decades, more attention has been paid to the histologic subtypes (16). For example, adenocarcinoma is the most common histology of lung cancer, representing almost half of lung cancer cases (17). Squamous cell carcinoma accounts for about $30 \%$ of NSCLC, which has a shorter doubling time, lower sensitivity to targeted therapy, and poorer survival than adenocarcinoma $(18,19)$. Other histologic subtypes, such as bronchioloalveolar carcinoma and carcinoid carcinoma, are rare but usually welldifferentiated and have high survival rates if resected at an early stage $(20,21)$. Given the different prognoses and responses to the treatment, it is important to determine the timeliness of treatment for each histologic subtype of lung cancer. In this study, we aimed to examine the association between diagnosis-to-surgery time interval and survival for stage I-IIA lung cancers and selected histologic subtypes. We present the following article in accordance with the STROBE reporting checklist (available at https://dx.doi. org/10.21037/tlcr-21-168).

\section{Methods}

\section{Data source and study population}

The Surveillance, Epidemiology, and End Results (SEER) program, which is supported by National Cancer Institute, collects cancer incidence data from populationbased cancer registries covering approximately $34.6 \%$ of the U.S. population (22). The SEER registries routinely collect data on patient demographics, primary tumor site, tumor morphology, stage at diagnosis, and first course of treatment. By linking with National Death Index and state death file, patients' vital status, date of last contact, and cause of death can be identified (22).

In this study, patients diagnosed with the American Joint Committee on Cancer (AJCC) stage I-IIA lung cancer during 2004 and 2015 (AJCC 6 ${ }^{\text {th }}$ Edition used for 2004-2009 cases and AJCC $7^{\text {th }}$ Edition used for 2010-2015 cases) were identified from the SEER database, including data from 18 cancer registries. Patients whose lung cancer was diagnosed as the only cancer or as the first primary cancer if more than one cancer were diagnosed, with negative lymph node involvement, receiving surgery of lobectomy, bilobectomy, or pneumonectomy, were included in the analyses. Exclusion criteria included: (I) patients with missing information on time interval between tumor diagnosis and treatment initiation, (II) patients receiving radiation before or before/after surgery or with sequence 
between radiation and surgery unknown; and (III) patients with less than one-month follow-up. All patients were followed until December 31, 2018.

All procedures performed in studies were in accordance with the Helsinki Declaration (as revised in 2013).

\section{Lung cancer histologic subtype}

Following the categorization proposed by SEER, lung cancer histologic subtype was determined based on the International Classification of Diseases for Oncology, $3^{\text {rd }}$ edition (ICD-O-3): adenocarcinoma (8140-8149), squamous or epidermoid carcinoma (8070-8079), bronchioloalveolar carcinoma (8250), large cell carcinoma (8012), adenosquamous carcinoma (8560), carcinoid (8240 8249), and small cell carcinoma (8041-8045) (23).

\section{Variables}

The primary exposure variable was the time interval between tumor diagnosis and surgery. The original variable in the SEER database was the interval between tumor diagnosis and treatment initiation (in months). We used this variable to approximate the interval between tumor diagnosis and surgery, as the initial treatment was highly likely to be surgery in this selected patient population. Three inclusion/exclusion criteria were applied to ensure the selected population receiving surgery as the initial treatment: (I) we included stage I-IIA lung cancer patients, for whom the recommended initial treatment is surgery if the cancer is operable (both NSCLC and SCLC) $(7,8)$; (II) we included only the patients who received a definitive surgery (lobectomy, bilobectomy, or pneumonectomy); and (III) we excluded the patients whose radiation therapy was given before the surgery or before/after the surgery and the patients whose sequence between radiation and surgery was unknown. In other words, stage I-IIA lung cancer patients who received surgery and the surgery was the only treatment or prior to radiation were included in the analyses, thus, we used the time interval between diagnosis and treatment initiation as a substitute for the time interval between diagnosis and surgery.

The time interval between tumor diagnosis and surgery was examined in a multi-categorical format: less than 1 month ( $<1$ month), 1 month or longer but less than 2 months (1-2 months), 2 months or longer but less than 3 months (2-3 months), 3 months or longer ( $\geq 3$ months). With multi-categorical variables, potential dose-dependent effect (longer delay, worse survival) can be detected by the trend analyses. Furthermore, to detect any non-linear associations (no trend for the worse survival associated with the increased time interval, but delay beyond a single cutoff point in the interval associated with worse survival) and maintain the sample size in multivariable analyses, especially for the less frequent histologic subtypes, a secondary exposure variable of the diagnosis-to-surgery interval was examined in a binary format: $\geq 1 v s .<1$ month, $\geq 2 v s$. $<2$ months, and $\geq 3$ vs. $<3$ months.

The outcome variables included lung cancer-specific survival and overall survival. In the analyses of lung-cancer specific survival, patients dying from other causes or alive at the end of follow-up were censored, and patients dying from unknown causes were excluded. Patients' survival time was calculated as the time interval between tumor diagnosis and date of last contact (death or end of the follow-up) in months.

Covariates included age at diagnosis $(<50,50-59,60-69$, $\geq 70$ years), sex, race (white, other), marital status (married, single or divorced or separated or widowed or unmarried or domestic partner, unknown), tumor size (AJCC tumor size T1, T2), grade (well differentiated, moderately differentiated, poorly differentiated or undifferentiated, unknown), histologic subtype, surgery type (resection of one lobe or bilobectomy, lobectomy or bilobectomy with dissection of lymph nodes or pneumonectomy), chemotherapy (yes, no or unknown), radiation therapy (yes, no or unknown), and study period (2004-2009, 2010-2015).

\section{Statistical analysis}

We used Chi-square tests to compare patient's sociodemographic and clinical characteristics. Kaplan-Meier survival curves were created to present the cancer-specific survival and overall survival distribution by diagnosisto-surgery interval, among all patients and stratified by histologic subtypes. Log-rank tests were conducted to examine the statistical differences in the Kaplan-Meier survival curves. We additionally applied Cox proportional hazards regressions to evaluate the survival differences associated with the diagnosis-to-surgery interval, with and without adjustment of covariates. In the analyses of the multi-categorical variable of diagnosis-to-surgery interval $(<1,1-2,2-3, \geq 3$ months), as $<1$ month was set as the reference group, the crude and adjusted odds ratio of each time interval compared to $<1$ month was calculated and reported. To examine the dose-dependent effect (whether 
increased time interval is associated with worse survival), $\mathrm{P}$ for trend was calculated by treating the time interval as a continuous variable. All analyses were carried out using SAS version 9.4 (SAS Institute, Cary, NC, USA) and statistical tests of significance were based on a two-sided test with significance levels of 0.05 .

\section{Results}

A total of 40,612 patients were analyzed in this study (Table 1), including $53.3 \%$ female and $84.8 \%$ white. Adenocarcinoma was the most frequent histologic subtype (40.1\%), followed by squamous or epidermoid carcinoma $(24.5 \%)$, and SCLC was the least frequent histologic subtype $(0.9 \%)$. The followup time ranged from 1 to 155 months, with a median of 51 months (interquartile 1-3: 26-87 months). During the follow-up, $39.8 \%$ of the patients died from any causes, while $23.1 \%$ of the patients died from cancer-specific causes.

The proportion of the patients receiving surgery $<1,1-2$, $2-3$, and $\geq 3$ months from diagnosis were $34.2 \%, 33.9 \%$, $19.8 \%$, and $12.1 \%$, respectively (Table 1 ). Younger, female, white, married patients and those with well-differentiated or T1 size tumor had shorter time interval to surgery, compared to their counterparts $(\mathrm{P}<0.0001)$. Within each histologic subtype, the proportion of patients receiving surgery within 1 month from diagnosis ranged from $28.9 \%$ for squamous or epidermoid carcinoma to $41.3 \%$ for bronchioloalveolar carcinoma; and the proportion of receiving surgery $\geq 3$ months after diagnosis ranged from $8.5 \%$ for SCLC to $13.9 \%$ for squamous or epidermoid carcinoma. Both the proportions of cancer-specific death (from $21.7 \%$ to $25.5 \%$ ) and all-cause death (from $37.5 \%$ to $44.8 \%$ ) increased as the diagnosis-to-surgery interval increased $(\mathrm{P}<0.0001)$.

Figure 1 shows the Kaplan-Meier cancer-specific survival curves by the time to surgery among all lung cancer patients and patients with each histologic subtype. Among all lung cancer patients, those receiving earlier surgery had better survival than those receiving later surgery $(\mathrm{P}<0.0001)$. Among adenocarcinoma patients, those with $<1$ month diagnosis-to-surgery interval had the best survival and those with $\geq 3$ months interval had the worst survival, while the survival curves for patients with intervals of 1-2 and 2-3 were greatly overlapped. For squamous or epidermoid carcinoma, except in the first 6 years of follow-up when $2-3$ and $\geq 3$ months to surgery showed similar survival, earlier surgery was associated with better survival. For bronchioloalveolar carcinoma, the cancer-specific survival was very similar in the first 5 years of follow-up regardless of time to surgery, but patients receiving surgery within 1 month from diagnosis had best survival during the rest of the follow-up time. Large cell lung cancer patients receiving surgery 3 months or later from diagnosis had worst survival than their counterparts who received earlier surgery. Time to surgery from diagnosis was not associated with cancer-specific survival for patients with adenosquamous carcinoma, carcinoid carcinoma, or small cell carcinoma.

Figure 2 shows the overall survival curves by the time interval between diagnosis and surgery. Similar to cancerspecific survival curves, earlier treatment was associated with better survival among all lung cancer patients and adenocarcinoma patients. For squamous or epidermoid carcinoma patients, those receiving surgery $\geq 3$ months had the worst survival, while $<1$ and 1-2 months diagnosisto-surgery interval had very similar survival curves. Early surgery showed better survival in bronchioloalveolar patients, except no obvious survival differences among those with $2-3$ and $\geq 3$ months intervals. No significant overall survival differences due to time to surgery were observed in patients with large cell carcinoma, adenosquamous carcinoma, carcinoid carcinoma, or small cell carcinoma.

Table 2 presents the results from Cox proportional hazards regressions, with and without adjusting covariates. Similar as shown in Kaplan-Meier survival curves, compared to lung cancer patients receiving surgery $<1$ month from diagnosis, delayed surgery was associated with $15 \%, 25 \%$, and $37 \%$ increased hazard of cancer-specific death for $1-2,2-3$, and $\geq 3$ months from diagnosis, respectively, without controlling for covariates $(\mathrm{P}<0.0001$ for each, $\mathrm{P}$ for trend $<0.0001)$. With adjustment of covariates, the increased hazard was 3\% [hazard ratio (HR): 1.03; $95 \%$ confidence interval (CI): 0.98-1.09], 10\% (HR: 1.10; 95\% CI: 1.04-1.16), and 22\% (HR: 1.22; 95\% CI: 1.14-1.30) for the three groups of patients, respectively ( $\mathrm{P}$ for trend $<0.0001)$. When using binary exposure variable, patients receiving surgery one month or later after diagnosis had 1.08 times the hazard of cancer-specific death (HR: 1.08; $95 \%$ CI: $1.04-1.13)$ than those receiving surgery within one month from diagnosis; $\geq 2$ months diagnosis-to-surgery interval was associated with 1.12 times the hazard of cancerspecific death compared to $<2$ month interval (HR: 1.12; 95\% CI: $1.07-1.17$ ); the HR for $\geq 3$ months interval was 1.17 (95\% CI: $1.10-1.25$ ) compared to $<3$ months interval. After stratified by histologic subtype, extended timeline to surgery was associated with worse cancer-specific survival and dose-dependent relationship was significant for 
Table 1 Characteristics of patients diagnosed with stage I-IIA lung cancer in Surveillance, Epidemiology, and End Results registries between 2004 and 2015

\begin{tabular}{|c|c|c|c|c|c|c|c|}
\hline Characteristics & \multicolumn{2}{|c|}{ All patients } & \multicolumn{5}{|c|}{ Time interval between diagnosis and treatment initiation (row \%) } \\
\hline All & 40,612 & & 34.2 & 33.9 & 19.8 & 12.1 & \\
\hline Age, years & & & & & & & $<0.0001$ \\
\hline $60-69$ & 14,427 & 35.5 & 35.2 & 33.6 & 19.0 & 12.2 & \\
\hline$\geq 70$ & 17,252 & 42.5 & 31.2 & 34.3 & 21.7 & 12.8 & \\
\hline Sex & & & & & & & $<0.0001$ \\
\hline White & 34,345 & 84.8 & 34.9 & 34.4 & 19.3 & 11.4 & \\
\hline Other & 6,160 & 15.2 & 30.3 & 30.8 & 22.5 & 16.5 & \\
\hline Marital status & & & & & & & $<0.0001$ \\
\hline Married & 23,430 & 57.7 & 35.4 & 35.6 & 19.0 & 9.9 & \\
\hline $\begin{array}{l}\text { Single, divorced, separated, widowed, } \\
\text { unmarried or domestic partner }\end{array}$ & 15,740 & 38.8 & 32.1 & 31.5 & 20.9 & 15.5 & \\
\hline Unknown & 1,442 & 3.55 & 38.7 & 30.7 & 19.8 & 11.6 & \\
\hline Tumor size & & & & & & & $<0.0001$ \\
\hline $\mathrm{T} 1$ & 22,018 & 54.2 & 39.0 & 31.4 & 18.1 & 11.6 & \\
\hline $\mathrm{T} 2$ & 18,594 & 45.8 & 28.6 & 36.8 & 21.8 & 12.8 & \\
\hline Surgery type & & & & & & & 0.0003 \\
\hline Resection of one lobe or bilobectomy & 8,906 & 21.9 & 36.0 & 32.6 & 19.1 & 12.2 & \\
\hline $\begin{array}{l}\text { Lobectomy or bilobectomy with dissection } \\
\text { of lymph nodes or pneumonectomy }\end{array}$ & 31,706 & 78.1 & 33.7 & 34.2 & 19.9 & 12.1 & \\
\hline Radiation therapy & & & & & & & 0.0002 \\
\hline Yes & 1,071 & 2.6 & 29.1 & 39.5 & 20.0 & 11.4 & \\
\hline No/unknown & 39,541 & 97.4 & 34.4 & 33.7 & 19.8 & 12.2 & \\
\hline
\end{tabular}

Table 1 (continued) 
Table 1 (continued)

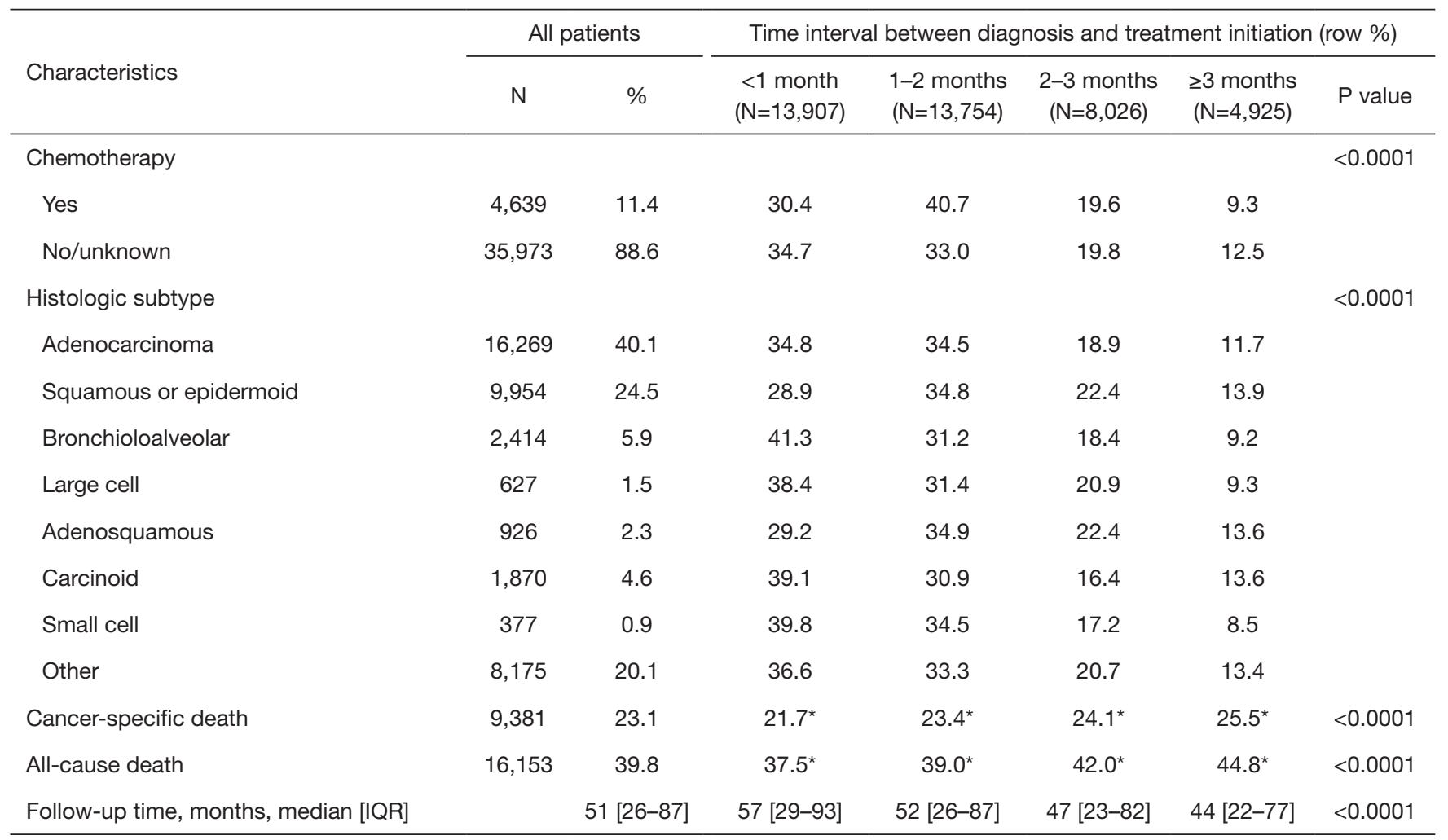

${ }^{*}$, proportion of death (\%) in each subgroup. IQR, inter-quartile range.

adenocarcinoma, squamous or epidermoid carcinoma, and bronchioloalveolar carcinoma. For large cell carcinoma, $>3$ month from diagnosis to surgery was associated with significant worse survival (HR: 1.63; 95\% CI: 1.09-2.44) than $<3$ months interval, controlling for covariates. No significant association was observed between time to surgery and cancer-specific survival for adenosquamous carcinoma, carcinoid carcinoma, and small cell carcinoma.

Table 3 shows the overall survival differences from Cox proportional hazards regressions. When using multi-categorical exposure variable of the diagnosisto-surgery interval, 2-3 and $\geq 3$ months were associated with significantly worse overall survival compared to $<1$ month for patients with any lung cancer, adenocarcinoma, squamous or epidermoid carcinoma, and bronchioloalveolar carcinoma ( $\mathrm{P}$ for trend $<0.0001$ for each), after adjusting for covariates. When using binary exposure variable, the longer time interval between diagnosis and surgery was associated with worse overall survival at each cut-off point for patients with any lung cancer, adenocarcinoma, squamous or epidermoid carcinoma, and bronchioloalveolar carcinoma.
No significant overall survival differences were observed for other histologic subtypes when adjusting for covariates.

\section{Discussion}

With a median follow-up of 51 months of a SEER retrospective cohort of 40,612 stage I-IIA lung cancer patients who received surgery, we examined the association between diagnosis-to-surgery time interval and the cancer-specific and overall survival. We found that extended diagnosis-to-surgery interval was associated with worse survival in all lung cancer patients and a few selected histologic subtypes. A strong dose-dependent effect from delayed surgery was detected in patients with adenocarcinoma and squamous or epidermoid carcinoma (longer delay, worse survival). No significant survival differences due to delayed surgery were observed among patients with adenosquamous carcinoma, carcinoid carcinoma, and small cell carcinoma. Findings on cancerspecific survival and overall survival were consistent.

In the past two decades, many studies examined the 

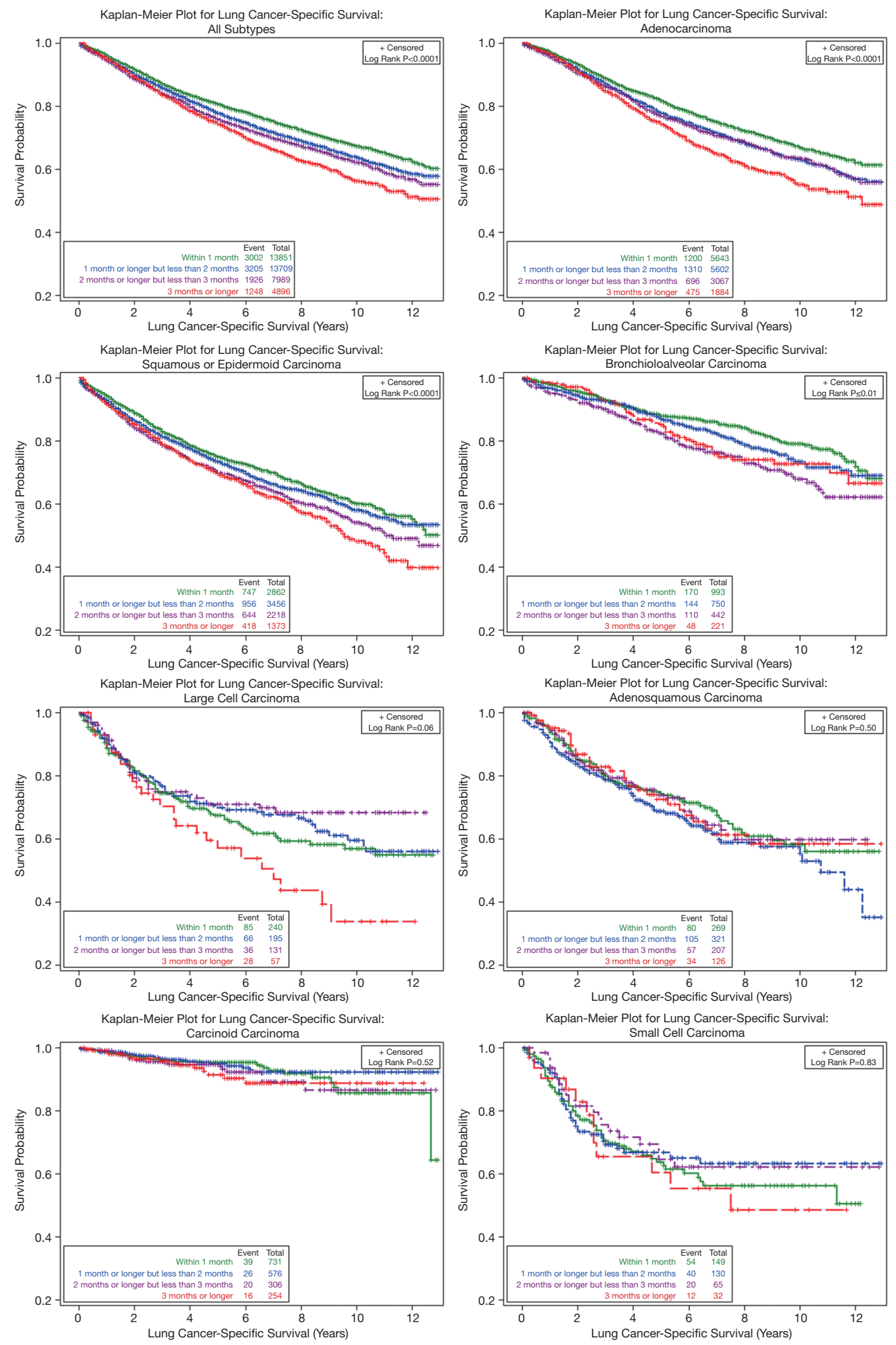

Figure 1 Kaplan-Meier plot of lung cancer-specific survival by time interval between cancer diagnosis and surgery. 

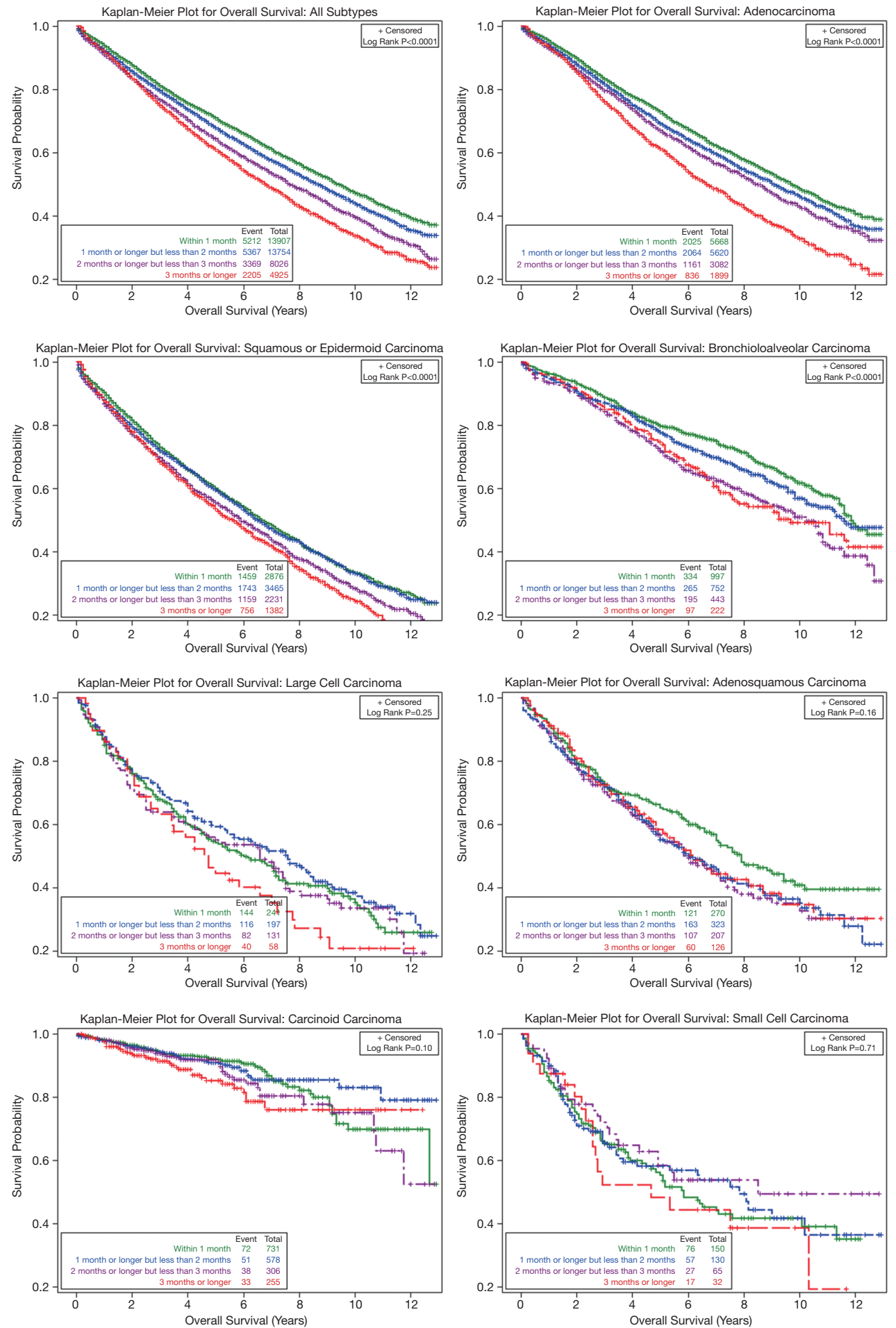

Figure 2 Kaplan-Meier plot of overall survival by time interval between cancer diagnosis and surgery. 
Table 2 Hazard ratios (95\% confidence intervals) of cancer-specific death by the time interval between lung cancer diagnosis and surgery, among all patients with stage I-IIA disease and stratified by histologic subtype

\begin{tabular}{|c|c|c|}
\hline Variable & Crude & Adjusted $^{*}$ \\
\hline $1-2$ vs. $<1$ month & $1.15(1.09-1.21)^{\Uparrow}$ & $1.03(0.98-1.09)$ \\
\hline $2-3$ vs. $<1$ month & $1.25(1.18-1.32)^{\Uparrow}$ & $1.10(1.04-1.16)^{\ddagger}$ \\
\hline$\geq 3$ vs. $<1$ month & $1.37(1.29-1.47)^{\Uparrow \S}$ & $1.22(1.14-1.30)^{1 \S}$ \\
\hline$\geq 2$ vs. $<2$ months & $1.21(1.16-1.26)^{\natural}$ & $1.12(1.07-1.17)^{\pi}$ \\
\hline$\geq 3$ vs. $<3$ months & $1.24(1.17-1.32)^{\Uparrow}$ & $1.17(1.10-1.25)^{\natural}$ \\
\hline \multicolumn{3}{|l|}{ Adenocarcinoma } \\
\hline $1-2$ vs. $<1$ month & $1.18(1.09-1.28)^{\pi}$ & $1.10(1.02-1.19)^{\dagger}$ \\
\hline$\geq 1$ vs. $<1$ month & $1.23(1.15-1.32)^{\Uparrow}$ & $1.13(1.06-1.22)^{\natural}$ \\
\hline$\geq 2$ vs. $<2$ months & $1.19(1.11-1.28)^{\Uparrow}$ & $1.12(1.04-1.20)^{\ddagger}$ \\
\hline$\geq 3$ vs. $<3$ months & $1.29(1.17-1.42)^{\Uparrow}$ & $1.22(1.11-1.34)^{\natural}$ \\
\hline \multicolumn{3}{|c|}{ Squamous or epidermoid carcinoma } \\
\hline $1-2$ vs. $<1$ month & $1.10(1.00-1.21)$ & $1.03(0.94-1.14)$ \\
\hline $2-3$ vs. $<1$ month & $1.24(1.12-1.38)^{\Uparrow}$ & $1.16(1.04-1.29)^{\ddagger}$ \\
\hline$\geq 3$ vs. $<1$ month & $1.32(1.17-1.48)^{\Uparrow \S}$ & $1.23(1.09-1.38)^{\ddagger \S}$ \\
\hline$\geq 1$ vs. $<1$ month & $1.18(1.09-1.29)^{\Uparrow}$ & $1.11(1.02-1.20)^{\dagger}$ \\
\hline \multicolumn{3}{|c|}{ Bronchioalveolar carcinoma } \\
\hline$\geq 3$ vs. $<1$ month & $1.39(1.01-1.91)^{\dagger \S}$ & $1.19(0.86-1.66)^{\|}$ \\
\hline$\geq 1$ vs. $<1$ month & $1.37(1.13-1.65)^{\ddagger}$ & $1.21(1.00-1.46)$ \\
\hline$\geq 2$ vs. $<2$ months & $1.42(1.17-1.72)^{\Uparrow}$ & $1.31(1.08-1.59)^{\ddagger}$ \\
\hline$\geq 3$ vs. $<3$ months & $1.17(0.87-1.57)$ & $1.07(0.79-1.44)$ \\
\hline \multicolumn{3}{|l|}{ Large cell carcinoma } \\
\hline $1-2$ vs. $<1$ month & $0.89(0.65-1.23)$ & $0.85(0.62-1.19)$ \\
\hline $2-3$ vs. $<1$ month & $0.76(0.52-1.13)$ & $0.65(0.43-0.96)^{\dagger}$ \\
\hline$\geq 3$ vs. $<1$ month & $1.46(0.95-2.23)$ & $1.40(0.91-2.15)$ \\
\hline$\geq 1$ vs. $<1$ month & $0.93(0.71-1.22)$ & $0.86(0.65-1.13)$ \\
\hline$\geq 2$ vs. $<2$ months & $1.01(0.76-1.36)$ & $0.91(0.68-1.23)$ \\
\hline$\geq 3$ vs. $<3$ months & $1.60(1.08-2.39)^{\dagger}$ & $1.63(1.09-2.44)^{\dagger}$ \\
\hline
\end{tabular}

Table 2 (continued) 
Table 2 (continued)

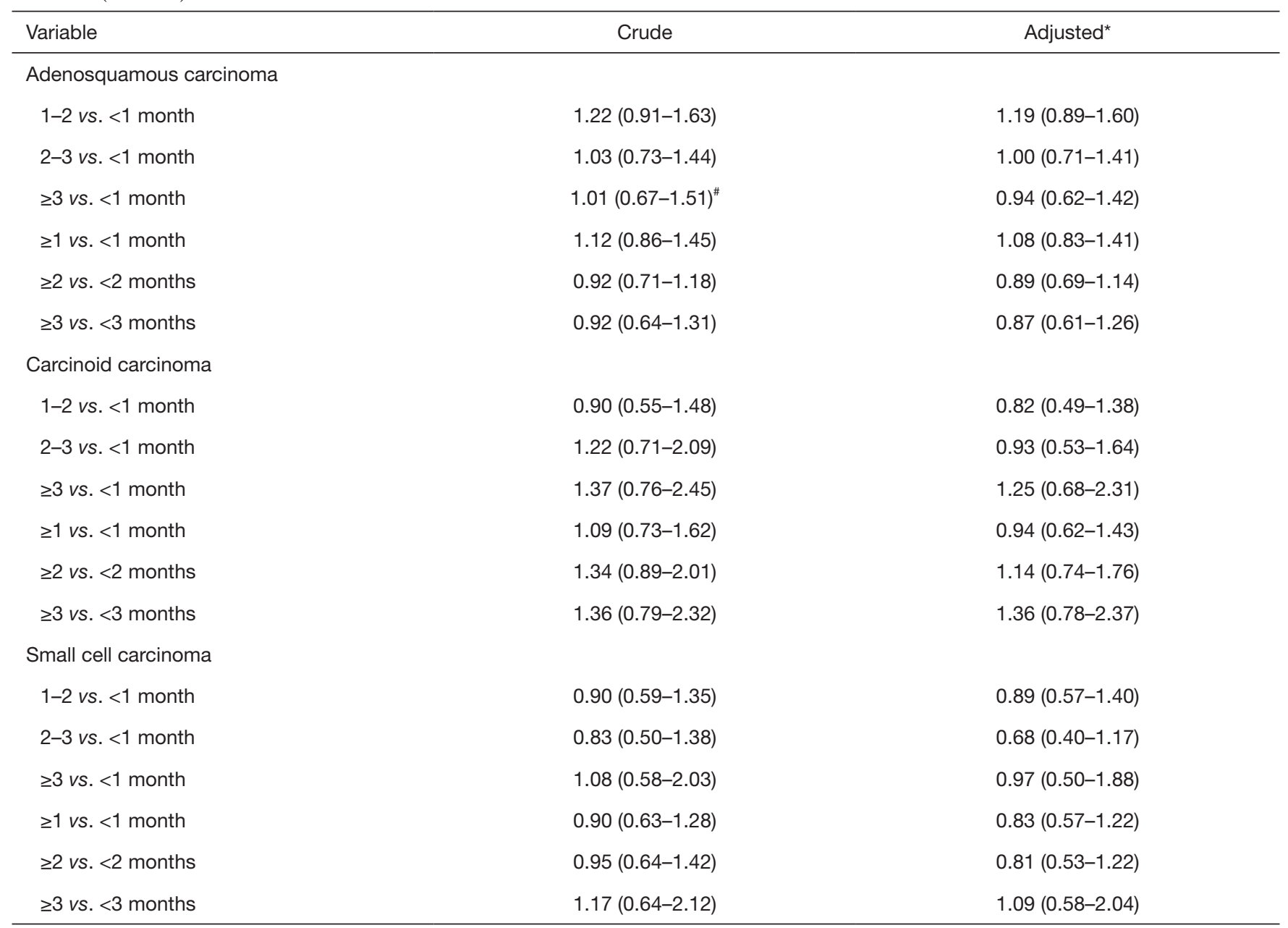

*, all model adjusted age at diagnosis, sex, race, marital status, tumor size, grade, surgery type, chemotherapy, radiation therapy, and study period. In the model for all patients, histologic subtype was also adjusted. ${ }^{\dagger}, \mathrm{P}<0.05 ;{ }^{\ddagger}, \mathrm{P}<0.01 ;{ }^{\natural}, \mathrm{P}<0.001$. ", $\mathrm{P}$ for trend $<0.05 ;{ }^{\prime}, \mathrm{P}$ for trend $<0.01 ;{ }^{\S}$, P for trend $<0.0001$.

correlation between timely care and lung cancer survival but yielded mixed and even paradoxical findings. Some studies reported better survival associated with timely diagnosis, staging, and treatment (24-27), some studies did not find any significant associations (28-31), while others found inverse associations $(32,33)$. Three possible reasons could explain the inconsistent findings. The first reason could be the unnoticed effect modification from stage and histology. Compared to advanced stage diseases, early stage diseases have a longer wait time due to multiple diagnostic tests and staging studies but better prognosis $(32,34)$. SCLC tends to have a shorter diagnosis-to-treatment interval but worse survival than NSCLC (32). Delayed diagnosis or treatment could impact survival differently across these patient subgroups. Without stratifying by these effect modifiers, the conclusions could be misleading. The second reason could be the different definitions of timely treatment used in the literature, given the inconsistent guidelines. The third reason could be the different treatment modalities used in the previous studies. In our study, we focused on the early stage disease treated with surgery and stratified the sample by histologic subtype to control the confounding and effect modification from stage and histology. In addition, we used the multi-categorical time interval as the exposure variable to examine the dose-dependent effect from delayed treatment, which addressed the concerns associated with binary analysis based on inconsistent guidelines.

There were two studies using the SEER-Medicare 
Table 3 Hazard ratios (95\% confidence intervals) of all-cause death by the time interval between lung cancer diagnosis and surgery, among all patients with stage I-IIA disease and stratified by histologic subtype

\begin{tabular}{|c|c|c|}
\hline Variable & Crude & Adjusted* \\
\hline $1-2$ vs. $<1$ month & $1.12(1.07-1.16)^{\pi}$ & $1.02(0.98-1.06)$ \\
\hline $2-3$ vs. $<1$ month & $1.28(1.22-1.33)^{\Uparrow}$ & $1.13(1.08-1.18)^{9}$ \\
\hline$\geq 3$ vs. $<1$ month & $1.43(1.36-1.50)^{\uparrow \S}$ & $1.24(1.18-1.31)^{1 \S}$ \\
\hline$\geq 2$ vs. $<2$ months & $1.26(1.22-1.30)^{9}$ & $1.16(1.12-1.20)^{9}$ \\
\hline$\geq 3$ vs. $<3$ months & $1.29(1.24-1.35)^{\Uparrow}$ & $1.20(1.15-1.26)^{\uparrow}$ \\
\hline \multicolumn{3}{|l|}{ Adenocarcinoma } \\
\hline $1-2$ vs. $<1$ month & $1.11(1.04-1.18)^{\Uparrow}$ & $1.05(0.99-1.12)$ \\
\hline$\geq 1$ vs. $<1$ month & $1.21(1.14-1.27)^{\natural}$ & $1.12(1.06-1.19)^{9}$ \\
\hline$\geq 2$ vs. $<2$ months & $1.26(1.19-1.33)^{\Uparrow}$ & $1.17(1.11-1.24)^{\Uparrow}$ \\
\hline$\geq 3$ vs. $<3$ months & $1.41(1.31-1.51)^{\Uparrow}$ & $1.30(1.21-1.40)^{9}$ \\
\hline \multicolumn{3}{|c|}{ Squamous or epidermoid carcinoma } \\
\hline $1-2$ vs. $<1$ month & $1.03(0.96-1.10)$ & $0.99(0.92-1.06)$ \\
\hline $2-3$ vs. $<1$ month & $1.17(1.08-1.26)^{\Uparrow}$ & $1.11(1.03-1.20)^{\dagger}$ \\
\hline$\geq 3$ vs. $<1$ month & $1.24(1.14-1.35)^{\text {१§ }}$ & $1.16(1.06-1.26)^{\ddagger \S}$ \\
\hline$\geq 1$ vs. $<1$ month & $1.11(1.04-1.18)^{\ddagger}$ & $1.06(0.99-1.12)$ \\
\hline \multicolumn{3}{|c|}{ Bronchioalveolar carcinoma } \\
\hline$\geq 3$ vs. $<1$ month & $1.42(1.14-1.79)^{\ddagger \S}$ & $1.29(1.03-1.62)^{\dagger \S}$ \\
\hline$\geq 1$ vs. $<1$ month & $1.29(1.13-1.48)^{\Uparrow}$ & $1.17(1.02-1.35)^{\dagger}$ \\
\hline$\geq 2$ vs. $<2$ months & $1.38(1.20-1.59)^{\natural}$ & $1.28(1.11-1.48)^{\natural}$ \\
\hline$\geq 3$ vs. $<3$ months & $1.25(1.01-1.55)^{\dagger}$ & $1.19(0.96-1.47)$ \\
\hline \multicolumn{3}{|l|}{ Large cell carcinoma } \\
\hline $1-2$ vs. $<1$ month & $0.89(0.70-1.14)$ & $0.89(0.69-1.14)$ \\
\hline $2-3$ vs. $<1$ month & $1.03(0.78-1.35)$ & $0.88(0.66-1.16)$ \\
\hline$\geq 3$ vs. $<1$ month & $1.28(0.90-1.82)$ & $1.21(0.85-1.72)$ \\
\hline$\geq 1$ vs. $<1$ month & $0.99(0.80-1.21)$ & $0.93(0.75-1.15)$ \\
\hline$\geq 2$ vs. $<2$ months & $1.16(0.93-1.44)$ & $1.02(0.82-1.27)$ \\
\hline$\geq 3$ vs. $<3$ months & $1.33(0.96-1.84)$ & $1.29(0.93-1.80)$ \\
\hline
\end{tabular}

Table 3 (continued) 
Table 3 (continued)

\begin{tabular}{|c|c|c|}
\hline Variable & Crude & Adjusted* \\
\hline $1-2$ vs. $<1$ month & $1.27(1.00-1.61)^{\dagger}$ & $1.21(0.95-1.53)$ \\
\hline $2-3$ vs. $<1$ month & $1.30(1.00-1.68)^{\dagger}$ & $1.24(0.95-1.62)$ \\
\hline$\geq 3$ vs. $<1$ month & $1.20(0.88-1.64)$ & $1.09(0.80-1.50)$ \\
\hline$\geq 2$ vs. $<2$ months & $1.11(0.91-1.34)$ & $1.07(0.88-1.30)$ \\
\hline$\geq 3$ vs. $<3$ months & $1.02(0.78-1.34)$ & $0.96(0.73-1.26)$ \\
\hline \multicolumn{3}{|l|}{ Carcinoid carcinoma } \\
\hline $1-2$ vs. $<1$ month & $0.96(0.67-1.37)$ & $0.95(0.66-1.37)$ \\
\hline$\geq 1$ vs. $<1$ month & $1.16(0.87-1.56)$ & $1.04(0.77-1.41)$ \\
\hline$\geq 2$ vs. $<2$ months & $1.40(1.05-1.88)^{\dagger}$ & $1.15(0.85-1.56)$ \\
\hline$\geq 3$ vs. $<3$ months & $1.50(1.03-2.18)^{\dagger}$ & $1.38(0.94-2.03)$ \\
\hline \multicolumn{3}{|l|}{ Small cell carcinoma } \\
\hline $1-2$ vs. $<1$ month & $0.94(0.67-1.33)$ & $0.96(0.66-1.39)$ \\
\hline $2-3$ vs. $<1$ month & $0.81(0.52-1.26)$ & $0.71(0.45-1.12)$ \\
\hline$\geq 3$ vs. $<1$ month & $1.13(0.67-1.91)$ & $1.06(0.61-1.85)$ \\
\hline$\geq 1$ vs. $<1$ month & $0.93(0.69-1.25)$ & $0.89(0.65-1.22)$ \\
\hline
\end{tabular}

*, all model adjusted age at diagnosis, sex, race, marital status, tumor size, grade, surgery type, chemotherapy, radiation therapy, and study period. In the model for all patients, histologic subtype was also adjusted. ${ }^{\dagger}, \mathrm{P}<0.05 ;{ }^{\ddagger}, \mathrm{P}<0.01 ;{ }^{\uparrow}, \mathrm{P}<0.001$. ${ }^{\#}, \mathrm{P}$ for trend $<0.05 ;{ }^{\prime}, \mathrm{P}$ for trend $<0.01 ;{ }^{\S}, \mathrm{P}$ for trend $<0.0001$.

dataset examining the consequences of delayed care on lung cancer survival. Both studies examined the survival effect of the time interval from diagnosis to any treatment initiation among all stage lung cancer patients. One study evaluated NSCLC patients from 2004-2007 and found that diagnosis-to-treatment interval $\leq 35$ days was associated with better survival among patients with localized disease, which was similar with our findings (34). Another study analyzed 2002-2007 NSCLC and SCLC patients but found a paradoxical association between timely treatment and overall survival (32). After stratified by stage, the association between timely care and worse survival was significant only among stage III \& IV patients, and there was a trend for a positive but insignificant association among stage I \&
II patients (32). Compared to these two studies, we used more contemporary data of curative patients and focused on the effect of timely surgery, i.e., stage I \& IIA patients who received definitive surgery. The more restricted study population can explain the higher prevalence of delayed treatment in our study (34\% of patients receiving treatment within 1 month from diagnosis in our study vs. higher than $60 \%$ of patients receiving timely care in the other two studies), as both early stage and receiving surgery are the risk factors of delayed treatment $(32,35)$. Among the studies focusing on the effect of timely surgery, two studies using National Cancer Data Base (NCDB) reported higher likelihood of pathologic upstaging and worse survival associated with delayed surgical resection among stage I 
NSCLC patients $(25,26)$, which was consistent with our findings. One study reported $4 \%$ increased hazard of death for every one week delay to surgery among stage I \& II community diagnosed NSCLC patients (36). Two studies analyzing stage I \& II NSCLC patients from single institution did not find significant association between delayed surgical resection and survival, for which the small sample size could be the reason $(29,37)$.

To our knowledge, our study is the first one examining the impact of delayed surgery on survival for each lung cancer histologic subtype. It was proposed that the faster growing tumors are more sensitive to extended wait time to treatment, as the longer diagnosis-to-treatment interval can result in worse outcomes due to the higher tumor growth rate (38). However, our data did not show this pattern. In our study, delayed surgery was associated with worse survival for adenocarcinoma, squamous cell carcinoma, bronchioloalveolar carcinoma, and large cell carcinoma, and the strength of the association was similar for these histologic subtypes. Similarly, another study found the survival effect of delay in any treatment was similar for NSCLC and SCLC, for all stages combined (32). One previous study analyzing a patient population with clinical stage IA lung squamous cell carcinoma from NCDB, reported $36 \%$ of patients receiving surgery within 30 days from diagnosis and 11-13\% increased risk of all-cause mortality associated with delayed surgery, which is very similar with our findings for squamous cell carcinoma (10-20\% increased risk) (39). Another recent study analyzing 286 propensity score matched clinical stage I lung adenocarcinoma patients found that delayed surgery ( $>21$ days from histologic diagnosis to surgery) is associated with two-fold risk of all-cause mortality $(\mathrm{HR}=2.03$, $\mathrm{P}=0.038$ ), which is higher than $10-36 \%$ increased risk in our adenocarcinoma patients. Additionally, for adenocarcinoma and squamous or epidermoid carcinoma, the longer interval between diagnosis and surgery was associated with worse cancer-specific and overall survival in our study ( $\mathrm{P}$ for trend $<0.0001)$.

No previous research investigated the effect of timely care on survival for other less frequent histologic subtypes of lung cancer. Our data was not able to detect significant survival differences associated with delayed surgery for adenosquamous carcinoma, carcinoid carcinoma, and SCLC, for which the smaller sample size could be a reason. Adenosquamous carcinoma contains both adenocarcinoma and squamous component, with each component constituting at least $10 \%$ of the tumor, and has higher tumor growth rate than adenocarcinoma (16). Our data showed that using 1 month as cutoff point to define delayed surgery tends to have stronger effect than using 3 months as cutoff point among adenosquamous carcinoma patients. Carcinoid carcinoma is a rare and well-differentiated neuroendocrine tumor (21), which had about $90 \%$ cancerspecific survival rate in our study. Undertaking surgery 3 months or later after diagnosis tended to be associated with worse cause-specific and overall survival for carcinoid carcinoma. Regarding SCLC, as majority of the diseases are inoperable at diagnosis in the clinical practice, for which the chemotherapy is the mainstay treatment, future studies with detailed information on the timing of other treatment are needed to determine the effect of timely care.

Our findings emphasize the clinical importance of timely care for lung cancer, which has been considered as a practice gap (40). In addition to survival benefit, timely care can relieve patients' stress and anxiety, and improve quality of life. However, multiple diagnostic tests and consultations could make the timely lung cancer management challenging. Previous studies examined the effects of nurseled care coordination (41), multidisciplinary clinics via telemedicine $(42)$, and outpatient diagnostic process $(43,44)$ to shorten the time to active treatment. More recent studies proposed the target time window to improve timely care $(34,36)$. Based on these findings, how to overcome the institutional challenges to provide timely management is a priority for lung cancer care.

Our study has several strengths. The first strength is the exclusive study population from a contemporary national database. We included only stage I-IIA patients who received surgery, which can avoid the confounding effect from stage and medical complexity (medically inoperable patients were excluded). Secondly, while most previous studies defined the delayed treatment into a single binary variable, we investigated the delayed treatment in both multi-categorical and binary format. By comparing the survival of patients receiving surgery $1-2,2-3$, and $\geq 3$ months to $<1$ month and providing $\mathrm{P}$ value for trend, we can evaluate the dose-dependent effect from delayed treatment, which can provide stronger evidence on the association. By using 1, 2, and 3 months as cutoff points to generate three binary variables, we have higher chance to identify the most appropriate interval for timely treatment for each histologic subtype. Thirdly, as majority of previous studies included only overall survival as the outcome, we additionally used cancer-specific survival as the outcome. Cancer-specific survival is a more sensitive indicator of the effect from cancer treatment and prognosis, especially for 
the old patient population.

Despite strengths, our study is also subjected to limitations. First of all, the exact date of surgery was not available in the original dataset. The time interval between the diagnosis and treatment initiation was used to approximate the diagnosis-to-surgery interval. Although we believe this approximation was appropriate as surgery was highly likely to be the initial treatment in the study population, future studies with exact surgery date are needed to confirm the findings. The second limitation is the lack of information on patient's comorbidity and performance status, which can be important confounders of timely surgery. We alleviated the confounding effect from comorbidity and performance status by including only patients who received surgery and using cancer-specific survival as an outcome. Patients with severe comorbid conditions or poor performance status who were not eligible for surgery were excluded in the analysis. Cancerspecific survival is less likely to be confounded by other health conditions and comorbidities. Thirdly, for the variables of chemotherapy utilization and radiation therapy utilization, no treatment and treatment unknown were grouped together. This is an inherent limitation of cancer registry data. The last limitation is the small sample size for less frequent histologic subtypes, especially SCLC. Larger sample size can provide more power to detect significant differences.

In summary, as the first study systematically examining the impact of delayed surgery on survival by histologic subtype, we found the delayed surgery is associated with worse survival in a large cohort of stage I-IIA lung cancer patients. The effect of delayed surgery was especially profound in adenocarcinoma, squamous or epidermoid carcinoma, bronchioloalveolar carcinoma, and large cell carcinoma. Our findings support the guidelines of receiving surgery within one month from tumor diagnosis in patients with stage I-IIA lung cancer. The observed dose-dependent effects emphasize the clinical importance of early treatment. Future studies with a larger sample size of less frequent histologic subtypes are warranted to provide more evidence for histology-specific lung cancer treatment guidelines.

\section{Acknowledgments}

Funding: This study was supported by Clemson University Internal Funding; National Cancer Institute's Surveillance, Epidemiology, and End Results (SEER) program (HHSN261201800007I/HHSN26100002 to XW); and the
National Program of Cancer Registries of the Centers for Disease Control and Prevention (1 NU58DP006332/ 5 NU58DP006332 to XW).

\section{Footnote}

Reporting Checklist: The authors have completed the STROBE reporting checklist. Available at https://dx.doi. org/10.21037/tlcr-21-168

Peer Review File: Available at https://dx.doi.org/10.21037/ tlcr-21-168

Conflicts of Interest: All authors have completed the ICMJE uniform disclosure form (available at https://dx.doi. org/10.21037/tlcr-21-168). The authors have no conflicts of interest to declare.

Ethical Statement: The authors are accountable for all aspects of the work in ensuring that questions related to the accuracy or integrity of any part of the work are appropriately investigated and resolved. All procedures performed in studies were in accordance with the Helsinki Declaration (as revised in 2013).

Open Access Statement: This is an Open Access article distributed in accordance with the Creative Commons Attribution-NonCommercial-NoDerivs 4.0 International License (CC BY-NC-ND 4.0), which permits the noncommercial replication and distribution of the article with the strict proviso that no changes or edits are made and the original work is properly cited (including links to both the formal publication through the relevant DOI and the license). See: https://creativecommons.org/licenses/by-nc-nd/4.0/.

\section{References}

1. Siegel RL, Miller KD, Jemal A. Cancer statistics, 2020. CA Cancer J Clin 2020;70:7-30.

2. Aberle DR, Adams AM, Berg CD, et al. Reduced lungcancer mortality with low-dose computed tomographic screening. N Engl J Med 2011;365:395-409.

3. Bleicher RJ, Ruth K, Sigurdson ER, et al. Time to Surgery and Breast Cancer Survival in the United States. JAMA Oncol 2016;2:330-9.

4. Hanna TP, King WD, Thibodeau S, et al. Mortality due to cancer treatment delay: systematic review and metaanalysis. BMJ 2020;371:m4087. 
5. Bagaria SP, Heckman MG, Diehl NN, et al. Delay to Colectomy and Survival for Patients Diagnosed with Colon Cancer. J Invest Surg 2019;32:350-7.

6. Liao CT, Chen HN, Wen YW, et al. Association between the diagnosis-to-treatment interval and overall survival in Taiwanese patients with oral cavity squamous cell carcinoma. Eur J Cancer 2017;72:226-34.

7. National Comprehensive Cancer Network. NCCN Clinical Practice Guidelines in Oncology: Non-small Cell Lung Cancer. Version 3, 2021.

8. National Comprehensive Cancer Network. NCCN Clinical Practice Guidelines in Oncology: Small Cell Lung Cancer. Version 3, 2021.

9. Rand Corp: Quality of Care for Oncologic Conditions and HIV: a Review of the Literature and Quality Indicators. 2020.

10. Alberts WM, Bepler G, Hazelton T, et al. Lung cancer. Practice organization. Chest 2003;123:332s-7s.

11. Ost DE, Jim Yeung SC, Tanoue LT, et al. Clinical and organizational factors in the initial evaluation of patients with lung cancer: Diagnosis and management of lung cancer, 3rd ed: American College of Chest Physicians evidence-based clinical practice guidelines. Chest 2013;143:e121S-e41S.

12. BTS recommendations to respiratory physicians for organising the care of patients with lung cancer. The Lung Cancer Working Party of the British Thoracic Society Standards of Care Committee. Thorax 1998;53 Suppl 1:S1-8.

13. National Institute for Health and Care Excellence: Clinical Guidelines. Lung cancer: diagnosis and management. London: National Institute for Health and Care Excellence (UK) Copyright (C NICE 2019.; 2019.

14. Jakobsen E, Green A, Oesterlind K, et al. Nationwide quality improvement in lung cancer care: the role of the Danish Lung Cancer Group and Registry. J Thorac Oncol 2013;8:1238-47.

15. van Meerbeeck JP, Fennell DA, De Ruysscher DK. Smallcell lung cancer. Lancet 2011;378:1741-55.

16. Travis WD, Brambilla E, Burke AP, Marx A, Nicholson AG. WHO Classification of Tumours of the Lung, Pleura, Thymus and Heart. Lyon: International Agency for Research on Cancer, 2015.

17. Travis WD, Brambilla E, Noguchi M, et al. International association for the study of lung cancer/American thoracic society/European respiratory society international multidisciplinary classification of lung adenocarcinoma. J Thorac Oncol 2011;6:244-85.
18. Heist RS, Sequist LV, Engelman JA. Genetic changes in squamous cell lung cancer: a review. J Thorac Oncol 2012;7:924-33.

19. Cheng TY, Cramb SM, Baade PD, et al. The International Epidemiology of Lung Cancer: Latest Trends, Disparities, and Tumor Characteristics. J Thorac Oncol 2016;11:1653-71.

20. Yousem SA, Beasley MB. Bronchioloalveolar carcinoma: a review of current concepts and evolving issues. Arch Pathol Lab Med 2007;131:1027-32.

21. Caplin ME, Baudin E, Ferolla P, et al. Pulmonary neuroendocrine (carcinoid) tumors: European Neuroendocrine Tumor Society expert consensus and recommendations for best practice for typical and atypical pulmonary carcinoids. Ann Oncol 2015;26:1604-20.

22. Surveillance, Epidemiology, and End Results Program. Available online: https://seer.cancer.gov/ Accessed on Jan. 12, 2021.

23. SEER ICD-O-3 Morphology Codes for Lung Cancer. Available online: https://training.seer.cancer.gov/lung/ abstract-code-stage/morphology.html Accessed on Jan 12, 2021.

24. Everitt S, Plumridge N, Herschtal A, et al. The impact of time between staging PET/CT and definitive chemoradiation on target volumes and survival in patients with non-small cell lung cancer. Radiother Oncol 2013;106:288-91.

25. Samson P, Patel A, Garrett T, et al. Effects of Delayed Surgical Resection on Short-Term and Long-Term Outcomes in Clinical Stage I Non-Small Cell Lung Cancer. Ann Thorac Surg 2015;99:1906-12; discussion 1913.

26. Bott MJ, Patel AP, Crabtree TD, et al. Pathologic Upstaging in Patients Undergoing Resection for Stage I Non-Small Cell Lung Cancer: Are There Modifiable Predictors? Ann Thorac Surg 2015;100:2048-53.

27. Saint-Jacques N, Rayson D, Al-Fayea T, et al. Waiting times in early-stage non-small cell lung cancer (NSCLC). J Thorac Oncol 2008;3:865-70.

28. Ha D, Ries AL, Montgrain P, et al. Time to treatment and survival in veterans with lung cancer eligible for curative intent therapy. Respir Med 2018;141:172-9.

29. Aragoneses FG, Moreno N, Leon P, et al. Influence of delays on survival in the surgical treatment of bronchogenic carcinoma. Lung Cancer 2002;36:59-63.

30. Vinod SK, Chandra A, Berthelsen A, et al. Does timeliness of care in Non-Small Cell Lung Cancer impact on survival? Lung Cancer 2017;112:16-24. 
31. Salomaa ER, Sällinen S, Hiekkanen H, et al. Delays in the diagnosis and treatment of lung cancer. Chest 2005;128:2282-8.

32. Nadpara P, Madhavan SS, Tworek C. Guidelineconcordant timely lung cancer care and prognosis among elderly patients in the United States: A population-based study. Cancer Epidemiol 2015;39:1136-44.

33. Myrdal G, Lambe M, Hillerdal G, et al. Effect of delays on prognosis in patients with non-small cell lung cancer. Thorax 2004;59:45-9.

34. Gomez DR, Liao KP, Swisher SG, et al. Time to treatment as a quality metric in lung cancer: Staging studies, time to treatment, and patient survival. Radiother Oncol 2015;115:257-63.

35. Maiga AW, Deppen SA, Pinkerman R, et al. Timeliness of Care and Lung Cancer Tumor-Stage Progression: How Long Can We Wait? Ann Thorac Surg 2017;104:1791-7.

36. Kanarek NF, Hooker CM, Mathieu L, et al. Survival after community diagnosis of early-stage non-small cell lung cancer. Am J Med 2014;127:443-9.

37. Quarterman RL, McMillan A, Ratcliffe MB, et al. Effect of preoperative delay on prognosis for patients with early stage non-small cell lung cancer. J Thorac Cardiovasc Surg

Cite this article as: Zhang $\mathrm{L}$, Hsieh MC, Rennert L, Neroda P, Wu XC, Hicks C, Wu J, Gimbel R. Diagnosis-to-surgery interval and survival for different histologies of stage I-IIA lung cancer. Transl Lung Cancer Res 2021;10(7):3043-3058. doi: 10.21037/tlcr-21-168
2003;125:108-13; discussion 113-4.

38. O'Rourke N, Edwards R. Lung cancer treatment waiting times and tumour growth. Clin Oncol (R Coll Radiol) 2000;12:141-4.

39. Yang CJ, Wang H, Kumar A, et al. Impact of Timing of Lobectomy on Survival for Clinical Stage IA Lung Squamous Cell Carcinoma. Chest 2017;152:1239-50.

40. Rankin NM, McGregor D, Stone E, et al. Evidencepractice gaps in lung cancer: A scoping review. Eur J Cancer Care (Engl) 2018;27:e12588.

41. Leary A, Corrigan P. Redesign of thoracic surgical services within a cancer network-using an oncology focus to inform change. Eur J Oncol Nurs 2005;9:74-8.

42. Davison AG, Eraut CD, Haque AS, et al. Telemedicine for multidisciplinary lung cancer meetings. J Telemed Telecare 2004;10:140-3.

43. Laroche C, Wells F, Coulden R, et al. Improving surgical resection rate in lung cancer. Thorax 1998;53:445-9.

44. Murray PV, O'Brien ME, Sayer R, et al. The pathway study: results of a pilot feasibility study in patients suspected of having lung carcinoma investigated in a conventional chest clinic setting compared to a centralised two-stop pathway. Lung Cancer 2003;42:283-90. 